\title{
Rickettsia raoultii sp. nov., a spotted fever group rickettsia associated with Dermacentor ticks in Europe and Russia
}

\author{
Oleg Mediannikov, ${ }^{1,2}$ Kotaro Matsumoto, ${ }^{1}$ Irina Samoylenko, ${ }^{3}$ \\ Michel Drancourt, ${ }^{1}$ Véronique Roux, ${ }^{1}$ Elena Rydkina, ${ }^{4}$ Bernard Davoust, ${ }^{5}$ \\ Irina Tarasevich, ${ }^{2}$ Philippe Brouqui ${ }^{1}$ and Pierre-Edouard Fournier ${ }^{1}$ \\ ${ }^{1}$ Unité des Rickettsies, CNRS UMR6020, IFR 48, Université de la Méditerranée, Faculté de \\ Médecine, 27 Blvd Jean Moulin, 13385 Marseille cedex 5, France \\ ${ }^{2}$ Gamaleya Research Institute of Epidemiology and Microbiology, Moscow, Russia \\ ${ }^{3}$ Omsk Research Institute of Natural Foci Infections, Omsk, Russia \\ ${ }^{4}$ Department of Medicine, University of Rochester School of Medicine and Dentistry, Rochester, NY, \\ USA \\ ${ }^{5}$ Direction Régionale du Service de Santé des Armées, 83800 Toulon Armées, France
}

Correspondence

Pierre-Edouard Fournier

Pierre-Edouard.Fournier @medecine.univ-mrs.fr
In 1999, three novel rickettsial genotypes, RpA4, DnS14 and DnS28, were identified in ticks collected in Russia using PCR amplification and sequencing of the rrs (16S rRNA), gltA and ompA genes (Rydkina et al., 1999). Rickettsia sp. genotypes DnS14 and DnS28 were detected in Dermacentor nuttalli ticks collected in Siberia, whereas genotype RpA4 was detected in Rhipicephalus pumilio ticks collected in Astrakhan (Rydkina et al., 1999). A later study demonstrated that Dermacentor ticks naturally infected with genotypes DnS14, DnS28 and RpA4 harbour these rickettsiae throughout the life cycle and that transovarial and transstadial transmission occurs (Samoilenko et al., 2003). These rickettsial agents form a reliable cluster within the Rickettsia massiliae group (Rydkina et al., 1999). This rickettsial group has been defined phylogenetically and phenotypically (Roux et al., 1996b; Rolain et al., 1998; Drancourt \& Raoult, 1999) and it consists of R. massiliae,

Abbreviations: MIF, microimmunofluorescence; SPD, specificity difference.

The GenBank/EMBL/DDBJ accession numbers for the 16S rRNA gene, gltA, ompA, ompB and sca4 sequences of strain $\mathrm{Khabarovsk}^{\top}$ are D0365810, DO365804, D0365801, D0365798 and DQ365808, respectively, and those of the $f t s Y$ and $r p o B$ gene sequences of strains Khabarovsk ${ }^{\top}$ and Marne are respectively D0387058 and D0387059 (fts $Y$ ) and D0365812 and D0365811 (rpoB). Accession numbers for sequences from other strains are detailed in Table 1.
Rickettsia rhipicephali, Rickettsia aeschlimannii and Rickettsia montanensis, which exhibit a unique feature within the genus Rickettsia, their resistance to rifampicin caused by a Phe-to-Leu mutation within the rpoB gene (Drancourt \& Raoult, 1999; Rolain et al., 1998). Due to their phylogenetic homogeneity, it was suggested that Rickettsia sp. genotypes DnS14, DnS28 and RpA4 belonged to a novel species (Rydkina et al., 1999). Genotypes identical to DnS14, DnS28 and RpA4 have also been detected in Dermacentor ticks from the European part of Russia (Shpynov et al., 2001, 2004), Spain (Ibarra et al., 2006) and Croatia (D. Raoult, personal communication). In 2002, we detected Rickettsia sp. genotype DnS14 by PCR in a Dermacentor marginatus tick taken from the scalp of a patient who had developed a typical clinical picture of tickborne lymphadenitis (TIBOLA) in France.

Herein, we describe the first cultivation of two rickettsial isolates genetically identical to Rickettsia sp. genotype DnS14 (Rydkina et al., 1999), two rickettsial isolates genetically identical to Rickettsia sp. genotype RpA4 (Rydkina et al., 1999) and one rickettsial isolate genetically identical to Rickettsia sp. genotype DnS28 (Rydkina et al., 1999). Using a polyphasic strategy combining genotypic and phenotypic tests, we demonstrate that these new rickettsial isolates fulfil the requirements for their classification within a novel species (Fournier et al., 2003; Raoult et al., 2005). 
Using cell culture (Marrero \& Raoult, 1989), we isolated five rickettsial strains from PCR-positive Dermacentor ticks collected on vegetation in Russia and France. Strain Khabarovsk $^{\mathrm{T}}$ was cultivated from a Dermacentor silvarum tick collected in Russian Far East in 2005, strain Shayman was obtained from a $D$. silvarum tick collected in eastern Siberia in 2002, strain Elanda-23/95 was cultivated from a D. nuttalli tick collected in Altay in 1995, strain Marne was cultivated from a Dermacentor reticulatus tick collected in the Marne region of eastern France in 2004 and strain 8/9 Karaganda was obtained from a Dermacentor marginatus tick collected in Kazakhstan in 2002. DNA was extracted from the five isolates using the QIAmp tissue kit (Qiagen) according to the manufacturer's instructions. Sequencing of the 16S rRNA, gltA, ompA, ompB and sca4 genes was attempted from all five isolates using previously described primers and PCR conditions (Roux \& Raoult, 1995, 2000; Roux et al., 1996a, 1997; Sekeyova et al., 2001). PCR products of the expected sizes were obtained from all five genes. Sequences were edited by removal of regions of ambiguity at the $5^{\prime}$ and $3^{\prime}$ ends so that their lengths were $1424,1134,590,4890$ and $3028 \mathrm{bp}$, respectively, for the $16 \mathrm{~S}$ rRNA gene, gltA, ompA, ompB and sca4. Accession numbers for these sequences are reported in Table 1 . Isolates Khabarovsk ${ }^{\mathrm{T}}$ and Shayman exhibited identical sequences and their 16S rRNA gene and gltA sequences were identical to those of Rickettsia sp. genotype DnS14 (Rydkina et al., 1999). Isolates Marne and 8/9 Karaganda also exhibited identical sequences and their 16S rRNA gene and $g l t A$ sequences were identical to those of Rickettsia sp. genotype RpA4 (Rydkina et al., 1999). Isolate Elanda-23/95 exhibited 16S rRNA gene and gltA sequences identical to those of Rickettsia sp. genotype DnS28 (Rydkina et al., 1999). The phylogenetic relationships of Rickettsia sp. strains Khabarovsk ${ }^{\mathrm{T}}$, Marne and Elanda-23/95 with all Rickettsia species with validly published names for which $16 \mathrm{~S}$ rRNA, gltA, ompA, ompB and sca4 gene sequences are available (Table 1) were evaluated for each gene using the neighbour-joining and maximum-parsimony methods within the MEGA 3.1 software (Kumar et al., 2004) and the maximum-likelihood method within the PHYLIP software package (Felsenstein, 1989). For all studied genes and using the three analysis methods, Rickettsia sp. strains Khabarovsk $^{\mathrm{T}}$, Marne and Elanda-23/95 clustered with the R. massiliae group with elevated bootstrap values (Fig. 1). When calculating similarity values between nucleotide sequences of strains Khabarovsk ${ }^{\mathrm{T}}$, Marne and Elanda-23/95 and Rickettsia species, transitions and transversions, not insertions or deletions, were included. For all five loci examined, strains Khabarovsk ${ }^{\mathrm{T}}$, Marne and Elanda-23/95

Table 1. GenBank accession numbers of the gene sequences used in the present study

\begin{tabular}{|c|c|c|c|c|c|}
\hline Strain & 16S rRNA gene & gltA & $o m p A\left(5^{\prime}\right.$ end $)$ & $o m p B$ & sca4 \\
\hline \multicolumn{6}{|l|}{ Rickettsia raoultii sp. nov. } \\
\hline Khabarovsk $^{\mathrm{T}}\left(=\right.$ CSUR R $\left.^{\mathrm{T}}\right)$ & DQ365810 & DQ365804 & DQ365801 & DQ365798 & DQ365808 \\
\hline Marne (=CSUR R9) & DQ365809 & DQ365803 & DQ365799 & DQ365797 & DQ365807 \\
\hline Elanda-23/95 (=CSUR R171) & EU036982 & EU036985 & EU036986 & EU036984 & EU036983 \\
\hline R. aeschlimannii $\mathrm{MC} 6^{\mathrm{T}}$ & U74757 & U59722 & U43800 & AF123705 & AF163005 \\
\hline R. africae ESF-5 & L36098 & U59733 & U43790 & AF123706 & AF151724 \\
\hline R. akari MK $(\text { Kaplan })^{\mathrm{T}}\left(=\right.$ ATCC VR-148 $\left.{ }^{\mathrm{T}}\right)$ & L36099 & U59717 & NA & AF123707 & AF213016 \\
\hline R. asiatica $\mathrm{IO}-1^{\mathrm{T}}\left(=\mathrm{CSUR} \mathrm{R} 2^{\mathrm{T}}\right)$ & AF394906 & AF394901 & NA & DQ110870 & DQ110869 \\
\hline R. australis NIAID Phillips $32^{\mathrm{T}}$ & L36101 & U59718 & AF149108 & AF123709 & AF187982 \\
\hline R. bellii 369L42-1 & L36103 & U59716 & NA & NA & $\mathrm{NA}$ \\
\hline R. canadensis $2678^{\mathrm{T}}\left(=\right.$ ATCC VR-610 $\left.{ }^{\mathrm{T}}\right)$ & L36104 & U59713 & NA & NA & $\mathrm{NA}$ \\
\hline R. conorii NIAID Malish $7^{\mathrm{T}}\left(=\right.$ ATCC VR-613 $\left.{ }^{\mathrm{T}}\right)$ & AF541999 & U59730 & U43806 & AF123721 & AF163008 \\
\hline R. felis URRWXCal2 (=ATCC VR-1525) & L28944 & AF210692 & AF210694 & AF210695 & AF196973 \\
\hline R. heilongjiangensis $054^{\mathrm{T}}\left(=\mathrm{ATCC} \mathrm{VR}-1524^{\mathrm{T}}\right)$ & AF178037 & AF178034 & AF179362 & AY260451 & AY331396 \\
\hline R. helvetica С9P9 & L36212 & U59723 & NA & AF123725 & AF163009 \\
\hline R. honei $\mathrm{RB}^{\mathrm{T}}\left(=\mathrm{ATCC} \mathrm{VR}-1472^{\mathrm{T}}\right)$ & U17645 & AF018074 & AF018075 & AF123711 & AF163004 \\
\hline R. japonica YM & L36213 & U59724 & U43795 & AF123713 & AF155055 \\
\hline R. massiliae Mtu1 ${ }^{\mathrm{T}}$ & L36214 & U59719 & U43799 & AF123714 & AF163003 \\
\hline R. montanensis $\mathrm{M} / 5-6$ & L36215 & U74756 & U43801 & AF123716 & AF163002 \\
\hline R. parkeri NIAID maculatum $20^{\mathrm{T}}$ & L36673 & U59732 & U43802 & AF123717 & AF155059 \\
\hline R. prowazekii Breinl ${ }^{\mathrm{T}}\left(=\mathrm{ATCC} \mathrm{VR}-142^{\mathrm{T}}\right)$ & M21789 & M17149 & NA & AF123718 & AF200340 \\
\hline R. rhipicephali Burgdorfer 3-7-female $6^{\mathrm{T}}$ & L36216 & U59721 & U43803 & AF123719 & AF155053 \\
\hline R. rickettsii $\mathrm{R}$ (Bitteroot) (=ATCC VR-891) & L36217 & U59729 & U43804 & X16353 & AF163000 \\
\hline R. sibirica $246^{\mathrm{T}}\left(=\mathrm{ATCC}\right.$ VR-151 $\left.{ }^{\mathrm{T}}\right)$ & L36218 & U59734 & U43807 & AF123722 & AF155057 \\
\hline R. slovaca $13-\mathrm{B}$ & L36224 & U59725 & $\mathrm{U} 43808$ & AF123723 & AF155054 \\
\hline R. tamurae AT- $1^{\mathrm{T}}\left(=\mathrm{CSUR} \mathrm{R} 1^{\mathrm{T}}\right)$ & AY049981 & AF394896 & DQ103259 & DQ113910 & DQ113911 \\
\hline R. typhi Wilmington ${ }^{\mathrm{T}}\left(=\right.$ ATCC VR-144 $\left.{ }^{\mathrm{T}}\right)$ & L36221 & U59714 & $\mathrm{NA}$ & L04661 & AF188482 \\
\hline
\end{tabular}

NA, Not amplifiable. 


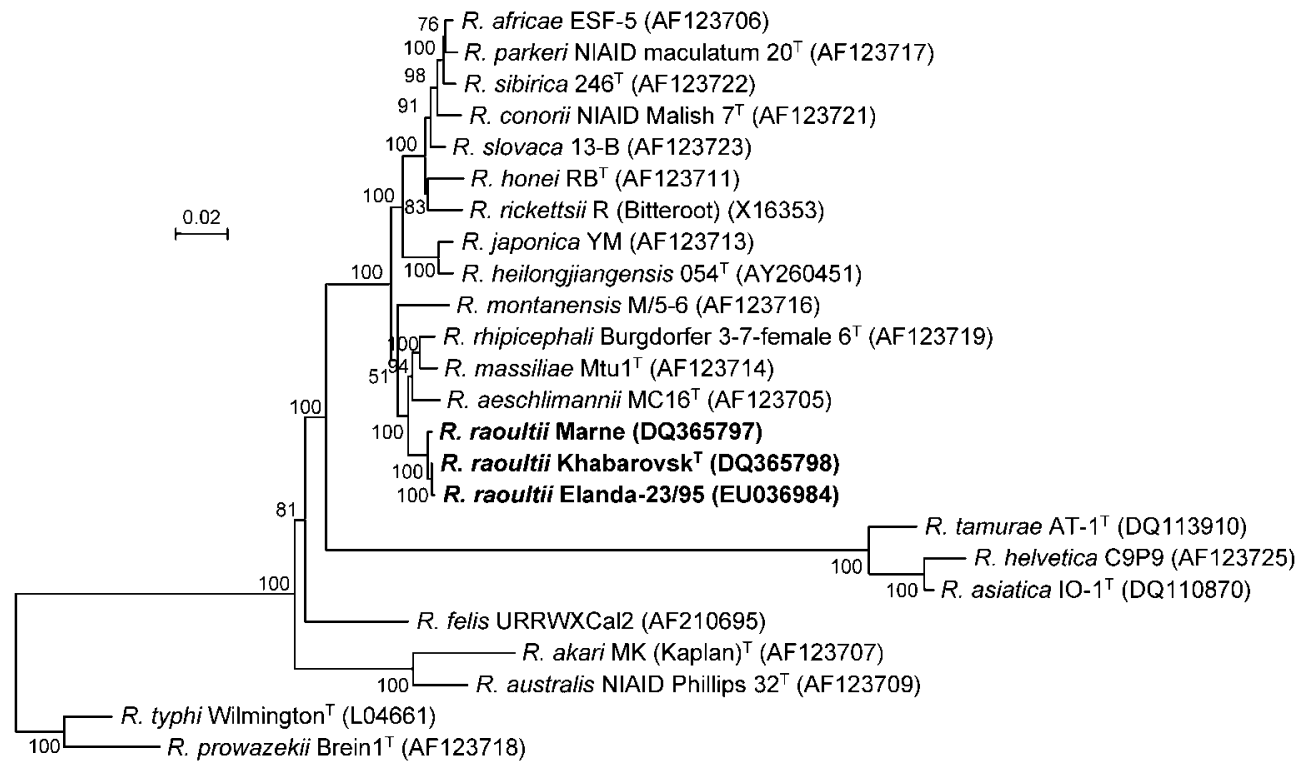

Fig. 1. Unrooted dendrogram showing the phylogenetic position of Rickettsia sp. isolates Khabarovsk ${ }^{\top}$, Marne and Elanda-23/ 95 among Rickettsia species inferred from the comparison of ompB sequences by the neighbour-joining method. Bootstrap values are indicated at nodes. Bar, $2 \%$ nucleotide sequence divergence.

shared highest sequence similarity with the type strain of $R$. rhipicephali (99.5, 99.2, 96.8, 98.1 and 97.7\%; 99.6, 99.1, 97.0, 98.1 and $97.9 \%$; and 99.5, 99.0, 96.6, 98.0 and $97.3 \%$, respectively, for the $16 \mathrm{~S}$ rRNA, gltA, ompA, ompB and sca 4 genes). However, these values were lower than the cut-offs proposed for Rickettsia species definition (Fournier et al., 2003). In contrast, similarity values higher than these cutoffs were found between strains Khabarovsk ${ }^{\mathrm{T}}$ and Marne (99.9, 99.9, 99.5, 99.6 and $99.5 \%)$, Khabarovsk ${ }^{\mathrm{T}}$ and Elanda-23/95 (99.9, 99.7, 99.5, 99.9 and 99.5\%) and Marne and Elanda-23/95 (99.9, 99.9, 99.3, 99.5 and 99.2\%). Therefore, on the basis of genotypic criteria, Rickettsia sp. strains Khabarovsk ${ }^{\mathrm{T}}$, Marne and Elanda-23/95, although closely related to members of the $R$. massiliae group (Fig. 1), belonged to a single distinct species. In addition, we compared Rickettsia sp. strains Khabarovsk ${ }^{\mathrm{T}}$, Marne and Elanda-23/95 to 'Rickettsia amblyommii' (Burgdorfer et al., 1981), also classified phylogenetically within the $R$. massiliae group. We found degrees of nucleotide sequence similarity of Rickettsia sp. strains Khabarovsk ${ }^{\mathrm{T}}$, Marne and Elanda-23/95 with ' $R$. amblyommii' of 99.1, 98.8, 97.3 and $96.3 \%, 99.3,98.8,97.1$ and $96.2 \%$ and $99.2,98.6,97.3$ and $96.0 \%$, respectively, for the $16 \mathrm{~S}$ rRNA, gltA, ompA and $o m p B$ genes, thus classifying it in a different species.

For further tests, we selected strains Khabarovsk ${ }^{\mathrm{T}}$ and Marne as representatives of this novel species. The $\mathrm{G}+\mathrm{C}$ contents of strains Khabarovsk ${ }^{\mathrm{T}}$ and Marne estimated by sequencing the $f t s Y$ gene as previously described (Fournier et al., 2006) were 33.9 and $33.8 \mathrm{~mol} \%$, respectively. By amplification and sequencing of a 364 bp rpoB fragment of Rickettsia sp. strains Khabarovsk ${ }^{\mathrm{T}}$ and Marne as described previously, we identified the mutation that confers rifampicin resistance in both strains previously identified in members of the R. massiliae group (Drancourt \& Raoult, 1999). Mouse serotyping was conducted by microimmunofluorescence (MIF) as described by Philip et al. (1978). We used as antigens Rickettsia sp. strains Khabarovsk ${ }^{\mathrm{T}}$ and Marne, R. rhipicephali Burgdorfer 3-7-female $6^{\mathrm{T}}, R$. aeschlimannii MC- $16^{\mathrm{T}}, R$. massiliae $\mathrm{Mtu}^{\mathrm{T}}$ and $R$. montanensis M/5-6, cultivated on L929 cells as described previously (Marrero \& Raoult, 1989). Strains Khabarovsk $^{T}$ and Marne caused cytopathic effects after 5 days of incubation. If the specificity difference (SPD) was $\geqslant 3$, the isolates were assumed to belong to different serotypes. Using serum from mice immunized with strain Khabarovsk ${ }^{\mathrm{T}}$, we found MIF antibody titres of $1: 512$ to the homologous antigen and to strain Marne and of $1: 256$ to the other four tested antigens. Using serum from mice immunized with strain Marne, we found MIF antibody titres of 1:512 to the homologous antigen and strain Khabarovsk $^{\mathrm{T}}$ and of $1: 128$ to the other four antigens. Serum from mice immunized with $R$. massiliae Mtu1 ${ }^{\mathrm{T}}$ produced antibody titres of $1: 1024$ to the homologous antigen and of 1:256 to strains Khabarovsk ${ }^{\mathrm{T}}$ and Marne. Serum from mice immunized with $R$. aeschlimannii MC$16^{\mathrm{T}}$ produced antibody titres of $1: 1024$ to the homologous antigen and of $1: 256$ to strains Khabarovsk ${ }^{\mathrm{T}}$ and Marne. Serum from mice immunized with $R$. rhipicephali Burgdorfer 3-7-female $6^{\mathrm{T}}$ produced antibody titres of $1: 1024$ to the homologous antigen and of $1: 256$ to strains Khabarovsk $^{\mathrm{T}}$ and Marne. Finally, using serum from mice immunized with $R$. montanensis M/5-6, we found antibody titres of $1: 256$ to the homologous antigen and of $1: 32$ to 
strains Khabarovsk $\mathrm{k}^{\mathrm{T}}$ and Marne. On the basis of these results, the SPD between these rickettsiae was 1, 4, 4, 3 and 4, respectively, between strain Khabarovsk ${ }^{\mathrm{T}}$ and strain Marne, $R$. massiliae, $R$. aeschlimannii, $R$. rhipicephali and $R$. montanensis. The SPD was $4,5,4$ and 3 , respectively, between strain Marne and $R$. massiliae, $R$. aeschlimannii, $R$. rhipicephali and $R$. montanensis. Therefore, the genotypic and serotypic specificity of Rickettsia sp. strains Khabarovsk ${ }^{\mathrm{T}}$ and Marne justify their classification within a distinct species. Using scanning microscopy, the cell length of isolate Khabarovsk $^{\mathrm{T}}$ varied from 0.6 to $2.0 \mu \mathrm{m}$ and the mean diameter was $0.4 \mu \mathrm{m}$ (Fig. 2a) and, using transmission electron microscopy, it was found free within the cytoplasm of L929 cells, but not in the nucleus (Fig. 2b).

Our data support the proposal to classify Rickettsia sp. strain Khabarovsk ${ }^{T}$ within a novel species. Thus, we formally propose the creation of Rickettsia raoultii sp. nov., which contains strains Khabarovsk ${ }^{\mathrm{T}}$, Marne, Shayman, 8/9 Karaganda and Elanda-23/95. This rickettsia has been found in France, Spain, Croatia, Russia and Kazakhstan.

\section{Description of Rickettsia raoultii sp. nov.}

Rickettsia raoultii (ra.oul'ti.i. N.L. masc. gen. n. raoultii of Raoult, named after Professor Didier Raoult, founder of
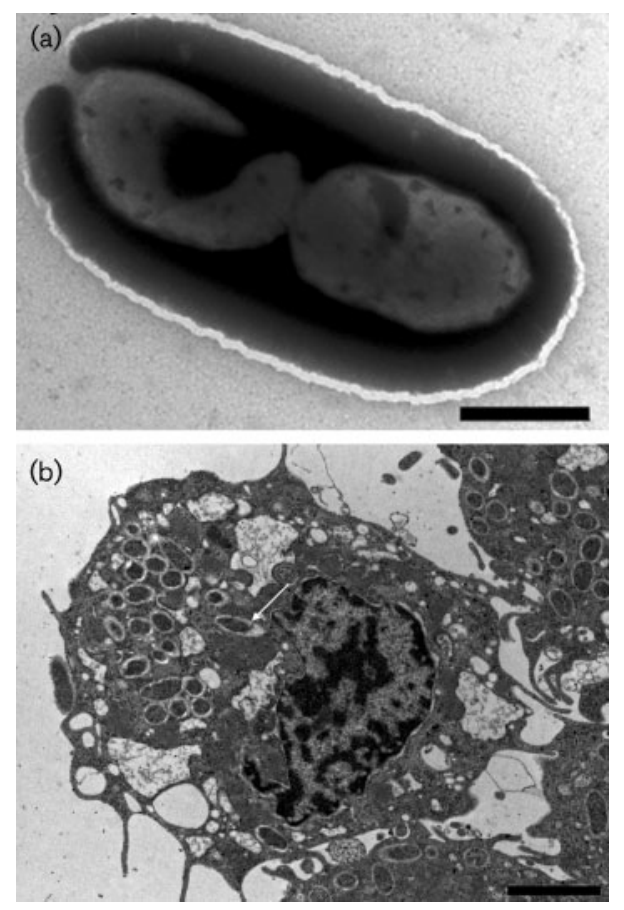

Fig. 2. (a) Rickettsia sp. isolate Khabarovsk ${ }^{\top}$ cultivated in L929 cells. Rickettsial bacillus in the process of division, with lophotrichous flagella. Scanning electron microscopy; bar, $1.0 \mu \mathrm{m}$. (b) Rickettsia sp. isolate Khabarovsk ${ }^{\top}$ appears as bacilli in the cytoplasm (arrow). Transmission electron microscopy; bar, $2.0 \mu \mathrm{m}$. the WHO-Collaborative Centre for Rickettsioses, Borrelioses and Tick-borne Infections in Marseilles, France, and a major contributor to the study of rickettsiae).

Gram-negative, obligately intracellular bacterium. Grows in $\mathrm{L} 929$ and Vero cells at $32{ }^{\circ} \mathrm{C}$ in minimal essential medium supplemented with $2 \%$ heat-inactivated fetal calf serum and $2 \mathrm{mM}$ glutamine. Non-motile. 16S rRNA, gltA, $o m p A, o m p B$ and sca4 gene sequencing indicate that this rickettsia is clearly different from all other recognized rickettsial species, the most closely related organism being $R$. massiliae. Estimated $\mathrm{G}+\mathrm{C}$ content is $33.8 \mathrm{~mol} \%$. Resistant to rifampicin. No information is available about the possible pathogenicity of this organism for vertebrate hosts. The known geographical distribution of this bacterium is France, Spain, Croatia, Russia and Kazakhstan.

The type strain is isolate Khabarovsk ${ }^{\mathrm{T}}\left(=\mathrm{CSUR} \mathrm{R}^{\mathrm{T}}\right.$ $=$ ATCC VR- $1596^{\mathrm{T}}$ ), which was isolated from Dermacentor silvarum ticks from the Russian Far East in 2005. The type strain has also been deposited in the rickettsial collection of the World Health Organization-Collaborative Gamaleya Institute under reference 147. Strains Shayman, Marne, 8/9 Karaganda and Elanda-23/95 have been deposited in the Collection de souches de l'unité des Rickettsies (CSUR), WHO-Collaborative Centre for Rickettsioses, Borrelioses and Tick-borne Infections, Marseille, France, under references CSUR R8, CSUR R9, CSUR R10 and CSUR R171, respectively, and are also being deposited in the ATCC.

\section{Acknowledgements}

The authors thank Guy Vestris for his valuable contribution to the culture of $R$. raoultii, Philippe Ulmer and Thierry Lamour for collecting ticks in eastern France and Leonid Ivanov for help with tick collection in the Russian Far East.

\section{References}

Burgdorfer, W., Hayes, S. F., Thomas, L. A. \& Lancaster, J. L. (1981). A new spotted fever group rickettsia from the lone star tick, Amblyomma americanum. In Rickettsiae and Rickettsial Diseases, pp. 595-602. Edited by W. Burgdorfer \& R. L. Anacker. New York: Academic Press.

Drancourt, M. \& Raoult, D. (1999). Characterization of mutations in the $r p o B$ gene in naturally rifampin-resistant Rickettsia species. Antimicrob Agents Chemother 43, 2400-2403.

Felsenstein, J. (1989). PHYLIP - phylogeny inference package (version 3.2). Cladistics 5, 164-166.

Fournier, P. E., Dumler, J. S., Greub, G., Zhang, J., Yimin, W. \& Raoult, D. (2003). Gene sequence-based criteria for the identification of new Rickettsia isolates and description of Rickettsia heilongjiangensis sp. nov. J Clin Microbiol 41, 5456-5465.

Fournier, P. E., Suhre, K., Fournous, G. \& Raoult, D. (2006). Estimation of prokaryote genomic DNA G + C content by sequencing universally conserved genes. Int J Syst Evol Microbiol 56, 1025-1029.

Ibarra, V., Oteo, J. A., Portillo, A., Santibáñez, S., Blanco, J. R., Metola, L., Eiros, J. M., Pérez-Martínez, L. \& Sanz, M. (2006). Rickettsia slovaca infection: DEBONEL/TIBOLA. Ann N Y Acad Sci 1078, 206-214. 
Kumar, S., Tamura, K. \& Nei, M. (2004). MEGA3: integrated software for molecular evolutionary genetics analysis and sequence alignment. Brief Bioinform 5, 150-163.

Marrero, M. \& Raoult, D. (1989). Centrifugation-shell vial technique for rapid detection of Mediterranean spotted fever rickettsia in blood culture. Am J Trop Med Hyg 40, 197-199.

Philip, R. N., Casper, E. A., Burgdorfer, W., Gerloff, R. K., Hugues, L. E. \& Bell, E. J. (1978). Serologic typing of rickettsiae of the spotted fever group by micro-immunofluorescence. J Immunol 121, 1961-1968.

Raoult, D., Fournier, P. E., Eremeeva, M., Graves, S., Kelly, P. J., Oteo, J. A., Sekeyova, Z., Tamura, A., Tarasevich, I. \& Zhang, L. (2005). Naming of rickettsiae and rickettsial diseases. Ann N Y Acad Sci 1063, $1-12$.

Rolain, J. M., Maurin, M., Vestris, G. \& Raoult, D. (1998). In vitro susceptibilities of 27 rickettsiae to 13 antimicrobials. Antimicrob Agents Chemother 42, 1537-1541.

Roux, V. \& Raoult, D. (1995). Phylogenetic analysis of the genus Rickettsia by $16 \mathrm{~S}$ rDNA sequencing. Res Microbiol 146, 385-396.

Roux, V. \& Raoult, D. (2000). Phylogenetic analysis of members of the genus Rickettsia using the gene encoding the outer-membrane protein rOmpB (ompB). Int J Syst Evol Microbiol 50, 1449-1455.

Roux, V., Fournier, P. E. \& Raoult, D. (1996a). Differentiation of spotted fever group rickettsiae by sequencing and analysis of restriction fragment length polymorphism of PCR amplified DNA of the gene encoding the protein rOmpA. J Clin Microbiol 34, 2058-2065.
Roux, V., Fournier, P. E., Rydkina, E. \& Raoult, D. (1996b) Phylogenetic study of the rickettsiae. In Rickettsiae and Rickettsial Diseases, pp. 34-42. Edited by J. Kazar \& R. Toman. Bratislava: Veda.

Roux, V., Rydkina, E., Eremeeva, M. \& Raoult, D. (1997). Citrate synthase gene comparison, a new tool for phylogenetic analysis, and its application for the rickettsiae. Int J Syst Bacteriol 47, 252-261.

Rydkina, E., Roux, V., Fetisova, N., Rudakov, N., Gafarova, M., Tarasevich, I. \& Raoult, D. (1999). New rickettsiae in ticks collected in territories of the former Soviet Union. Emerg Infect Dis 5, 811-814.

Samoilenko, I. E., Rudakov, N. V., Shpynov, S. N., Tankibaev, M. A., Yakimenko, V. V. \& Kumpan, L. V. (2003). Study of biological characteristics of spotted fever group rickettsial genotypes RpA4, DnS14, and DnS28. Ann N Y Acad Sci 990, 612-616.

Sekeyova, Z., Roux, V. \& Raoult, D. (2001). Phylogeny of Rickettsia spp. inferred by comparing sequences of 'gene D', which encodes an intracytoplasmic protein. Int J Syst Evol Microbiol 51, 1353-1360.

Shpynov, S., Parola, P., Rudakov, N., Samoilenko, I., Tankibaev, M., Tarasevich, I. \& Raoult, D. (2001). Detection and identification of spotted fever group rickettsiae in Dermacentor ticks from Russia and central Kazakhstan. Eur J Clin Microbiol Infect Dis 20, 903-905.

Shpynov, S., Fournier, P. E., Rudakov, N., Tankibaev, M., Tarasevich, I. \& Raoult, D. (2004). Detection of a rickettsia closely related to Rickettsia aeschlimannii, "Rickettsia heilongjiangensis", Rickettsia sp. strain RpA4, and Ehrlichia muris in ticks collected in Russia and Kazakhstan. J Clin Microbiol 42, 2221-2223. 